\title{
RISCOS BIOLÓGICOS OCUPACIONAIS EM EQUIPE DE ATENDIMENTO PRÉ-HOSPITALAR DO CORPO DE BOMBEIROS DE PERNAMBUCO
}

\section{RESUMO}

\author{
Robson Marques da Silva \\ Gabriela Cunha Schechtman Sette ${ }^{2}$ \\ Telma Marques da Silva \\ Jadiane Ingrid da Silva ${ }^{4}$ \\ Ana Paula Esmeraldo Lima ${ }^{5}$
}

O estudo objetivou investigar os riscos ocupacionais biológicos entre profissionais do Atendimento Pré-Hospitalar Móvel do Corpo de Bombeiros de Pernambuco. Estudo transversal, descritivo, quantitativo, com 163 Bombeiros Militares. Utilizou-se para a coleta de dados um instrumento semiestruturado sobre questões de exposição a materiais biológicos potencialmente contaminados, comportamentos e condutas. A prevalência de exposição aos materiais biológicos foi de $73 \%$. Destes, $87,1 \%$ usavam algum equipamento de proteção individual, $96,64 \%$ não realizaram o Atestado de Origem e $74,8 \%$ negligenciaram aconselhamento médico pós-exposição. $O$ sangue foi 0 principal agente de contato e $16,6 \%$ dos casos ocorreram durante procedimentos de imobilizações. $49,1 \%$ desconhecem normas preventivas no grupamento e metade dos participantes tem imunização incompleta. A presente pesquisa possibilitou concluir que os bombeiros militares estão expostos a riscos ocupacionais em suas atividades laborais de assistência, sobretudo aos riscos biológicos. Considera-se pertinente, melhores orientações aos profissionais quanto à relevância de condutas preventivas, mudanças de comportamentos pós-exposição aos Materiais Biológicos Potencialmente Contaminado se adesão adequada à imunização.

Palavras-chave: Exposição ocupacional; Agentes biológicos; Atendimento préhospitalar; Saúde do trabalhador.

\footnotetext{
${ }^{1}$ Graduado em Enfermagem pela Universidade Federal de Pernambuco (UFPE). Bombeiro Militar. Email: robson_marques163@hotmail.com

${ }^{2}$ Enfermeira. Doutora em Saúde da Criança e do Adolescente da UFPE. Professora Adjunto da UFPE.

${ }^{3}$ Enfermeira. Doutora pela Universidade Federal de Santa Catarina. Professora Associado da UFPE.

${ }^{4}$ Enfermeira. Mestranda do Programa de Pós-Graduação em Enfermagem pela UFPE

${ }^{5}$ Enfermeira. Doutora em Saúde da Criança e do Adolescente da UFPE. Professora Adjunto da UFPE.
} 


\title{
OCCUPATIONAL BIOLOGICAL RISKS ON PROFESSIONALS FROM THE PRE-HOSPITAL MOBILE SERVICE OF THE FIRE DEPARTMENT OF PERNAMBUCO
}

\begin{abstract}
The study aimed to investigate the risk of biological occupational on professionals from the Pre-Hospital Mobile Service of the Fire Department of Pernambuco. This cross-sectional, descriptive, and quantitative study included 163 firefighters. The data was collected using a semi-structured instrument on exposure issues to Potentially Contaminated Biological Materials, and associated behaviors and conducts. The prevalence of exposure to biological materials was $73 \% .87 .1 \%$ of these were composed of firefighters who used some type of personal protective equipment, $96.64 \%$, of firefighters who did not take the Origin Certificate, and $74.8 \%$, of firefighters who neglected medical advices after the exposure. Blood was the main contact agent and $16.6 \%$ of the cases occurred during the immobilization procedures. $49.1 \%$ of the participants do not know preventive norms, and half of them have incomplete immunization. Based on this research, it was possible to conclude that firefighters are exposed to occupational hazards in their work activities, especially biological ones. It is considered appropriate to provide better guidance to professionals regarding the relevance of preventive behaviors, behavior changes after post Potentially Contaminated Biological Materials exposure, and adequate adherence to immunization.
\end{abstract}

Keywords: Occupational exposure; Biological factors; Emergency medical services; Occupational health.

Artigo Recebido em 21/12/2018 - Aceito em 22/03/2019 - Publicado em 31/03/2019 


\section{INTRODUÇÃO}

Atendimento Pré-Hospitalar (APH) é todo atendimento assistencial no ambiente fora da unidade de saúde, a vítimas de agravos de natureza clínica, traumática ou psíquica, que possam levar a sofrimentos, sequelas ou mesmo à morte, sendo necessário, portanto, prestar-Ihe atendimento e/ou transporte adequado, visando a manutenção da vida e minimização dos danos, através de um serviço de saúde devidamente regulado e qualificado (DAL PAl et al., 2015).

No Brasil, o serviço prestado pelo APH é realizado pelo Serviço de Atendimento Móvel de Urgência (SAMU) e pelo Corpo de Bombeiro Militar (CBM). O primeiro é composto por profissionais com formação e atuação na área da saúde, como médicos, enfermeiros e técnicos de enfermagem, podendo atuar tanto nas unidades de suporte básico de vida (SBV) quanto nas de suporte avançado de vida (BRASIL, 2014).

O CBM, por sua vez, possui profissionais com treinamento em SBV, podendo ou não ter formação complementar na área da saúde, o que impossibilita a realização de procedimentos complexos e/ou invasivos, como por exemplo a punção venosa para reposição volêmica. Normalmente desempenha ações de salvamento e socorro público, estabilizações de veículos acidentados, gerenciamento e monitoramento de risco quando solicitados, retirada de vítimas de locais de difícil acesso ou promoção de acesso a equipes de saúde para a vítima, administração de oxigênio, ventilação e circulação artificial por meio das compressões torácicas em reanimação cardiopulmonar (RCP), ações de educação preventiva contra acidentes, dentre outros (DAL PAl et al., 2015; BRASIL, 2014).

Os serviços prestados pelo SAMU e CBM seguem as diretrizes e regulamentações do Ministério da Saúde, com gerência e financiamento do Sistema Único de Saúde (SUS), ofertado de forma gratuita aos usuários. A 
assistência do APH otimiza tratamento, reduz o tempo de hospitalização e traz melhor prognóstico de reabilitação (BRASIL, 2014).

Considerando a peculiaridade dos atendimentos prestados e do ambiente extra-hospitalar, geralmente de difícil controle de riscos, os profissionais que atuam no APH estão expostos a uma variedade de riscos ocupacionais, independentemente de sua formação. Entende-se por riscos ocupacionais toda e qualquer situação ou circunstância existentes na atividade realizada por um profissional em um determinado serviço ou ambiente, que têm o potencial de causar ou aumentar algum dano ao seu bem-estar físico ou psíquico (OLIVEIRA; PAIVA, 2013; ALUKO et al., 2016).

Dentre estes riscos, destacam-se os biológicos, por constituírem a principal forma de exposição do profissional, quando da manipulação direta ou indireta de material biológico, resultante da assistência à saúde. Os materiais biológicos potencialmente contaminados (MBPC) são provenientes de fluidos corporais (saliva, vômitos, lágrimas, expectoração, urina, fezes e sangue) e têm a capacidade de transmitir doenças quando em contato direto com pele e mucosas(OLIVEIRA; PAIVA, 2013).

Os agentes biológicos que representam um risco ocupacional são todos os micro e macro organismos, e as substâncias produzidas por eles, que têm um efeito adverso sobre os seres humanos no processo de trabalho. Muitas vezes apresentam-se de forma imperceptível aos profissionais, pela falta de conhecimento ou informação técnica a respeitos destes males (ARAÚJO; MOREIRA, 2015; BREWCZYŃSKA et al., 2015).

As dificuldades de controle em zonas quentes (local onde se encontra a vítima/local da ocorrência), a não adesão do uso correto e completo dos equipamentos de proteção individuais (EPI) e a falta de conhecimento prévio do histórico de saúde da vítima, constituem alguns dos riscos à saúde destes trabalhadores em cada assistência(OLIVEIRA; PAIVA, 2013). As dificuldades presentes no $\mathrm{APH}$, tornam o serviço incomum e por vezes hostil, com risco da 
própria vida destes profissionais, pela existência dos riscos identificados ou não (ABREU et al., 2016).

Estudo realizado com o Corpo de Bombeiros de Minas Gerais evidenciou que $53,3 \%$ dos profissionais do APH tiveram exposição a materiais biológicos em suas assistências, e metade dos casos ocorreu por contato em mucosas (oral ou ocular). Identificou-se ainda que mais de um terço dos acidentes com MBPC aconteceram mais de uma vez, alertando para a vulnerabilidade dos Bombeiros Militares (BM) do APH aos riscos biológicos e doenças ocupacionais (OLIVEIRA, MACHADO, GAMA, 2014).

Outro estudo também realizado em Minas Gerais, com a equipe de enfermagem do APH do SAMU, demonstrou que $83,3 \%$ da população estudada já sofreu algum tipo de acidente com exposição aos MBPC. Observou-se que a maior ocorrência desse tipo de acidente acontece quando desempenham as manobras de resgaste. Apenas 6,7\% dos acidentes ocorreram por falta de atenção, negligência ou imprudência, indicando que a atividade do APH deixa o profissional iminentemente exposto aos riscos ocupacionais em todas as suas atuações (LEITE et al., 2016).

Diante do exposto, despertou-se a necessidade de responder à seguinte questão: Qual a situação dos riscos biológicos ocupacionais entre os profissionais do Grupamento de Bombeiros do Atendimento Pré-Hospitalar de Pernambuco (GBAPH)? Assim, o presente estudo teve como objetivo investigar os riscos ocupacionais biológicos entre profissionais do Atendimento PréHospitalar Móvel do Corpo de Bombeiros de Pernambuco. Espera-se que os resultados desta pesquisa possam ser usados em medidas de prevenção de acidentes de trabalho, assim como na promoção de instrução, treinamento e qualificação dos profissionais bombeiros.

\section{MÉTODO}


Estudo do tipo transversal, descritivo, com abordagem quantitativa. A pesquisa foi realizada no GBAPH Móvel do Estado de Pernambuco, com sede em Olinda, Prazeres, São Lourenço da Mata e Igarassu, no período de fevereiro a junho de 2018.

A população foi composta por $246 \mathrm{BM}$ do GBAPH de PE. O tamanho amostral foi calculado utilizando-se o programa Epitable no software Epi Info ${ }^{\mathrm{TM}}$ 7 , considerando-se erro amostral de 5\%, nível de significância de $95 \%$, frequência do registro do evento de 58\% (TRIPPLE et al., 2013) e 10\% para possíveis perdas, totalizando $163 \mathrm{BM}$. A seleção da amostra foi por conveniência, sendo incluídos os BM que atuavam no GBAPH há no mínimo um ano e excluídos aqueles que estavam de folga, licença de qualquer natureza ou férias.

A coleta de dados foi realizada mediante a assinatura do Termo de Consentimento e Livre Esclarecimento, no próprio local de trabalho dos BM. Haja vista a natureza de atendimento de urgência/emergência prestada pelos referidos profissionais, a fim de viabilizar a coleta e interromper o mínimo possível suas atividades, os instrumentos eram entregues aos profissionais e recolhidos após preenchimento, em horário mais conveniente para os participantes.

Foi utilizado um questionário semiestruturado, adaptado (SOERENSEN, 2008), composto por questões quanto à caracterização do profissional GBAPH e ocorrência de exposição a MBPC, com as seguintes variáveis de estudo: idade, sexo, secção que servi no GBAPH, tempo de serviço, atestado de origem, os riscos biológicos ocupacionais, uso de equipamentos de proteção individuais, situação vacinal (imunoproteção), formação em saúde e possíveis ocorrências que envolveram MBPC, suas características e condutas pósevento.

Os dados foram digitados e processados no Statistical Package for the Social Sciences (SPSS, versão 21.0) mediante análise estatística descritiva, das frequências relativa e absoluta, assim como da média como medida de 
Revista Científica do Corpo de Bombeiros Militar de Pernambuco

Artigo publicado no Vol.05 º12 - Edição de JAN a JUN 2019 - ISSN 2359-4829

Versão on-line disponível em: http://www.revistaflammae.com.

tendência central. A pesquisa seguiu os preceitos da Resolução n 466/2012 do Conselho Nacional de Saúde, sendo aprovada pelo Comitê de Ética em Pesquisa, do Centro de Ciências da Saúde da Universidade Federal de Pernambuco, sob o parecer de $\mathrm{N}^{\circ} 2.175 .150$.

\section{RESULTADOS}

Dos 163 participantes, 148(90,8\%) eram do sexo masculino. A idade variou entre 29 a 53 anos, com média de 41 anos. Os profissionais atuavam nos seguintes locais: 99 (61\%) no auto-resgate, 33 (20\%) no administrativo, 21 (13\%) na moto resgate e $10(6 \%)$ em outros (estafeta, expurgo, almoxarifado, manutenção e graduado de dia). Nos GBAPH onde ocorreram as coletas, houve maior número de participantes nas sedes de Olinda 58 (36\%), Prazeres 36 (22\%) e São Lourenço 28 (17\%). A maioria dos profissionais 115 (70,5\%) possuía de 6 a 10 anos de serviço no GBAPH.

Sobre os MBPC, $119(73 \%)$ participantes afirmaram ter sofrido exposição no $\mathrm{APH}$, mas apenas $4(2,5 \%)$ realizaram Atestado de Origem (AO) pósexposição. $81(49,7 \%)$ informaram o contato direto em mais de uma região corporal e $56(34,4 \%)$ referiram os membros superiores como parte mais afetada. 83(50,9\%) confirmaram que a assistência no APH expõe o profissional ao MBPC, sendo a imobilização identificada como o principal procedimento de exposição, representando $16,6 \%$ (27). O cenário de maior ocorrência da exposição aos MBPC foi a zona quente $68(41,7 \%)$, e o sangue foi o principal fluido corporal de exposição, com 80(49,1\%) (Tabela 1).

Quando expostos aos MBPC, 142(87,1\%) relataram estar fazendo uso de algum EPI no APH. Apenas 51 (14,05\%) relataram o uso de todos os EPI (luvas, máscaras e óculos) em suas assistências. A maioria dos profissionais, $122(74,8 \%)$, negligenciou a consulta médica após o contato com MBPC (Tabela 1). 
Revista Científica do Corpo de Bombeiros Militar de Pernambuco

Artigo publicado no Vol.05 Nº12 - Edição de JAN a JUN 2019 - ISSN 2359-4829

Versão on-line disponível em: http://www.revistaflammae.com.

Tabela 1 - Caracterização da exposição à MBPC dos BM do

GBAPH. Pernambuco, 2018

\begin{tabular}{|c|c|c|}
\hline Variáveis & $\mathbf{N}$ & $\%$ \\
\hline Entrevistados & 163 & 100 \\
\hline \multicolumn{3}{|l|}{ Exposição a MBPC } \\
\hline Sim & 119 & 73 \\
\hline Não & 43 & 26,4 \\
\hline Não responderam & 1 & 0,6 \\
\hline \multicolumn{3}{|l|}{ Região corporal de contato MBPC } \\
\hline Face & 1 & 0,6 \\
\hline Boca & 2 & 1,2 \\
\hline Braço & 37 & 22,7 \\
\hline Mão & 19 & 11,7 \\
\hline Perna & 2 & 1,2 \\
\hline Mais de uma região corporal & 81 & 49,7 \\
\hline Não responderam & 21 & 12,9 \\
\hline \multicolumn{3}{|c|}{ Assistência no APH de exposição MBPC } \\
\hline Entrevista & 1 & 0,6 \\
\hline Exame físico & 4 & 2,5 \\
\hline Colocação de vítima na prancha & 9 & 5,5 \\
\hline Colocação de colar cervical & 2 & 1,2 \\
\hline Oxigenioterapia & 4 & 2,5 \\
\hline Imobilização & 27 & 16,6 \\
\hline Extricação de vítimas & 5 & 3,1 \\
\hline Todas assistências citadas & 83 & 50,9 \\
\hline Não responderam & 19 & 11,7 \\
\hline Outras & 9 & 5,5 \\
\hline \multicolumn{3}{|c|}{ Cenários de ocorrência da exposição } \\
\hline Zona quente & 68 & 41,7 \\
\hline Ambulância & 7 & 4,3 \\
\hline Desinfeccão & 4 & 2,5 \\
\hline Mais de um cenário & 63 & 38,7 \\
\hline Não responderam & 21 & 12,8 \\
\hline \multicolumn{3}{|l|}{ MBPC exposto } \\
\hline Saliva & 1 & 0,6 \\
\hline Vômito & 1 & 0,6 \\
\hline Expectoração & 11 & 6,7 \\
\hline Sangue & 80 & 49,1 \\
\hline Mais de um MBPC & 59 & 36,2 \\
\hline Não responderam & 11 & 6,7 \\
\hline \multicolumn{3}{|c|}{ Uso de EPI quando em contato MB } \\
\hline Sim & 142 & 87,1 \\
\hline Não & 4 & 2,5 \\
\hline Não responderam & 17 & 10,4 \\
\hline \multicolumn{3}{|l|}{ Desinfecção pós-exposição } \\
\hline Álcool 70\% & 74 & 45,4 \\
\hline Sabão + álcool 70\% & 48 & 29,4 \\
\hline Não responderam & 19 & 11,7 \\
\hline Outros & 22 & 13,5 \\
\hline \multicolumn{3}{|l|}{ Consulta médica pós-exposicão } \\
\hline Sim & 20 & 12,3 \\
\hline Não & 122 & 74,8 \\
\hline
\end{tabular}


Revista Científica do Corpo de Bombeiros Militar de Pernambuco

Artigo publicado no Vol.05 Nº12 - Edição de JAN a JUN 2019 - ISSN 2359-4829

Versão on-line disponível em: http://www.revistaflammae.com.

\begin{tabular}{lcc}
\hline Não responderam & 21 & 12,9 \\
Normas de Prevenção no GBAPH & 80 & 49,1 \\
Sim & 49 & 30,1 \\
Não & 3 & 1,8 \\
Não responderam & 31 & 19 \\
Não sei & & \\
Atividade exposição MBPC & 7 & 4,3 \\
Sobrecarga de trabalho & 11 & 6,7 \\
Operações de resgate & 6 & 3,7 \\
Distração & 7 & 4,3 \\
Materiais biológicos nas atuações & 15 & 9,2 \\
Outras & 108 & 66,3 \\
Todas atividades do APH & 9 & 5,5 \\
Não responderam & 363 & 100 \\
EPI de maior utilização no APH & 138 & 38,02 \\
Luvas procedimentos & 6 & 1,65 \\
Luvas de tecido & 10 & 2,75 \\
Luvas couro/raspa & 71 & 19,56 \\
Óculos & 66 & 18,18 \\
Máscara & 6 & 1,65 \\
Outros & 1 & 0,28 \\
Não usava & 51 & 14,05 \\
Usavam todos EPI básicos & &
\end{tabular}

A Tabela 2, que trata da situação vacinal e formação em saúde dos BM, apresenta que apenas $49,7 \%(81)$ dos participantes possuíam cartões de vacinação atualizados e 50,3\% (82) estavam passíveis a doenças pela falta de imunização. Quanto à proteção imunológica contra vírus da Hepatite B, 72,4\% (118) dos bombeiros referiram ter o esquema completo de vacinação.22,7\%(37) dos bombeiros militares possuíam formação ou eram estudantes da área da saúde, dos quais mais da metade eram profissionais de enfermagem (Enfermeiros, Técnico e Auxiliares de enfermagem). 
Revista Científica do Corpo de Bombeiros Militar de Pernambuco

Artigo publicado no Vol.05 Nº12 - Edição de JAN a JUN 2019 - ISSN 2359-4829

Versão on-line disponível em: http://www.revistaflammae.com.

Tabela 2. Situação vacinal e formação em saúde dos

BM do GBAPH. Pernambuco, 2018

\begin{tabular}{lcc}
\hline Variáveis & $\mathbf{N}$ & $\%$ \\
\hline Entrevistados & $\mathbf{1 6 3}$ & $\mathbf{1 0 0}$ \\
\hline Cartão de Vacina atualizado & 81 & 49,7 \\
Sim & 75 & 46 \\
Não & 5 & 3,1 \\
Não sei & 2 & 1,2 \\
Não responderam & & \\
Participação nas campanhas de & 115 & \\
vacinação & 2 & 70,6 \\
Sim & 46 & 1,2 \\
Não & 118 & 28,2 \\
Não responderam & 10 & \\
Vacinas contra Hepatite - B (HVB) & 35 & 72,4 \\
Sim & 37 & 6,1 \\
Não & 115 & 21,5 \\
Não responderam & 11 & 22,7 \\
Formação em saúde no GBAPH & & 70,6 \\
Sim & 2 & 6,7 \\
Não & 14 & 5,4 \\
Não responderam & 3 & 37,8 \\
Categoria Profissional & 10 & 8,1 \\
Auxiliar de enfermagem & 8 & 27 \\
Técnico de enfermagem & Bacharel em Enfermagem & 21,6 \\
Estudante de cursos de saúde & Outras áreas de saúde &
\end{tabular}

Fonte: os autores

*Categoria Profissional considerada para os BM que têm formação em saúde ( $\mathrm{n}=37)$

\section{DISCUSSÃO}

A pesquisa apresentou um alto índice de exposição a MBPC pelos bombeiros, evidenciando um perfil de risco de morbidade destes profissionais ao contato com agentes biológicos, o que evidencia a importância de uma vigilância em saúde da população trabalhadora do CBM (GUIMARÃES; FELLI, 2016). Constata-se no GBAPH de Pernambuco a alta prevalência de exposição dos profissionais com os MBPC, mesmo não realizando procedimentos invasivos (SOUSA, SOUZA, COSTA, 2014).

As peculiaridades do APH potencializam a exposição dos profissionais aos MBPC em suas assistências, aumentando a vulnerabilidade dos BM a diversos tipos de doenças ocupacionais (MARTINS et al., 2014). Dessa forma é 
importante o amplo conhecimento destes técnicos sobre os riscos que estão enfrentando para melhor prevenção (LORO, 2016).

A maioria relatou a não elaboração do Atestado de Origem sobre os acidentes com MBPC, apresentando como justificativa não achar necessário informar tal evento ou não ter conhecimento da necessidade da comunicação ao Oficial de Área responsável que estivesse de plantão no dia do ocorrido. Uma grande problemática dos agravos acometidos aos profissionais na assistência, é a falta de notificação, que impossibilita medidas precoce de prevenção e acompanhamento do estado de saúde deste profissional pósevento(GUIMARÃES; FELLI, 2016).

O principal ambiente relatado para a exposição aos MBPC foi a zona quente, local característico por ser muitas vezes inusitado, de difícil controle e monitoramento. A vulnerabilidade ocupacional dos BM, portanto, ameaça a prestação de serviços de saúde de qualidade.

O sangue foi o principal fluido corporal de exposição, mas mais de um terço dos profissionais relataram contato com mais de um tipo de material biológico.Regiões corpóreas não íntegras em contato com MBPC, poderão veicular doenças. Os acidentes com MBPC podem ser por inoculação percutânea, provocada por lesão com objetos contaminados e por contato direto pele e/ou mucosa na assistência do $\mathrm{APH}$, podendo trazer danos à saúde destes profissionais quando estes fluídos estão contaminados(OLIVEIRA, MACHADO, GAMA, 2014).

A maioria dos profissionais negligenciaram a procura de consulta médica e condutas pós-exposição, desconsiderando os possíveis danos a sua saúde e de sua equipe. Há a importância de reforçar as condutas profiláticas nas primeiras duas horas pós-exposição, como coleta de sangue, quimio profilaxias e acompanhamento clínico dos envolvidos. Os procedimentos de prevenção de acidentes com MBPC e de pós-exposição, precisam ser de conhecimento de todos, pois norteiam condutas a serem realizadas, que possam prevenir a 
exposição ou minimizar qualquer dano à saúde dos profissionais. (OLIVEIRA, MACHADO, GAMA, 2014; ARAÚJO; SILVA, 2014; PIMENTA et al., 2013).

$O$ alto risco biológico ocupacional verificado nesse estudo poderia ser minimizado se os EPI preconizados fossem utilizados nos atendimentos, o que não foi constatado. As medidas de proteção restringiram-se predominantemente ao uso das luvas de procedimento, dados semelhantes aos encontrados em estudo realizado no CBM do APH de Santa Catarina (RUFATTO, 2011).

As medidas de Precaução-Padrão são importantes para prevenir a aquisição de doenças como a hepatite $B$, hepatite $C$, vírus da imunodeficiência adquirida (HIV), sífilis, doença de chagas e influenza, e devem ser adotadas sempre que houver risco de contato com sangue ou outros fluidos corpóreos. Consiste, entre outros, no uso dos seguintes EPI: luva, avental ou uniforme impermeável com manga, óculos e máscara(RUFATTO, 2011; ARAÚJO; SILVA, 2014).

No Brasil, o uso dos EPI é regulamentado pela NR6, que exige seu uso pelo profissional bem como seu fornecimento pelas instituições (BRASIL, 2009). Apesar da obrigatoriedade da sua utilização e do elevado risco de contato com agentes biológicos, a adesão ao uso dos EPI é baixa no APH, conforme constatado nesse estudo, contribuindo para a exposição dos profissionais aos riscos ocupacionais.

Observou-se que entre os profissionais existe uma parcela significativa de BM não vacinados, o que não se constatou naqueles com formação na área da saúde. No entanto, estudo realizado na Nigéria identificou que apenas $64,2 \%$ dos profissionais da saúde apresentavam imunização completa contra Hepatite B, uma das principais doenças ocupacionais infecciosas (ALUKO et al., 2016)

Todo trabalhador voltado à assistência em saúde deve estar imunologicamente protegido, não só contra a hepatite $B$, mas contra todas as doenças imunopreveníveis. A disponibilização das vacinações nas UBS constitui uma forma preventiva que impede a disseminação dessas 
Revista Científica do Corpo de Bombeiros Militar de Pernambuco

Artigo publicado no Vol.05 º12 - Edição de JAN a JUN 2019 - ISSN 2359-4829

Versão on-line disponível em: http://www.revistaflammae.com.

doenças(OLIVEIRA, MACHADO, GAMA, 2014; SOCIEDADE BRASILEIRA DE IMUNIZAÇÕES, 2018).A capacitação técnica e superior de profissionais pode colaborar para a diminuição da exposição aos riscos ocupacionais que possa causar danos a sua saúde em situações de urgência e emergência (OLIVEIRA, SANTOS, SANTOS, 2013).

\section{CONCLUSÃO}

A presente pesquisa possibilitou identificar que os BM do GBAPH estão expostos a riscos ocupacionais em suas atividades laborais de assistência, sobretudo aos riscos biológicos. Houve alta prevalência à exposição de MBPC, sobretudo nos cenários de zona quente, sendo mais frequente quando a assistência era prestada durante o procedimento de imobilização. O sangue foi o material biológico de maior exposição e poucos bombeiros utilizavam os EPI recomendados para assistência, embora alguns tenham formação profissional na área da saúde, em especial na enfermagem. Além disso, os riscos ainda podem ser agravados decorrentes da cobertura vacinal incompleta e ausência de condutas profiláticas pós-exposição, o que atenuaria a ocorrência de doenças imunopreveníveis e ocupacionais.

Diante destes resultados, recomenda-se a adesão de ações e comportamentos seguros, além da realização de educação permanente sobre a temática de prevenção de acidentes biológicos, que garantam a qualidade da assistência e promoção da saúde dos BM do GBAPH de PE.

\section{REFERÊNCIAS}

ABREU, G. S. A. et al.Riscos biológicos e o Atendimento Pré-Hospitalar. Revista Interdisciplinar em Saúde, Cajazeiras,v. 3, n. 1, p.179-196, 2016.

ALUKO, O. O. et al. Knowledge, attitudes and perceptions of occupational hazards and safety practices in Nigerian healthcare workers. BMC research notes, v. 9, n. 1, p. 71-85, 2016. 


\section{Revista FLAMMAE}

Revista Científica do Corpo de Bombeiros Militar de Pernambuco

Artigo publicado no Vol.05 No12 - Edição de JAN a JUN 2019 - ISSN 2359-4829

Versão on-line disponível em: http://www.revistaflammae.com.

ARAÚJO, L. R.A., MOREIRA, M. R. Risco ocupacional enfrentado pela equipe de enfermagem do serviço de atendimento móvel de urgência.Revista Científica Eletrônica da FAIT, v. 2, n. 3, 2015.

ARAÚJO, T. M. E.; SILVA, N. C. Acidentes perfuro cortantes e medidas preventivas para hepatite $B$ adotadas por profissionais de Enfermagem nos serviços de urgência e emergência de Teresina, Piauí. Revista Brasileira de Saúde Ocupacional, v. 39, n. 130, 2014.

BRASIL. Ministério da Saúde. Secretaria de Atenção à Saúde. Departamento de Atenção Protocolos de Intervenção para o SAMU 192 - Serviço de Atendimento Móvel de Urgência.Brasília: Ministério da Saúde, 2014, 236p.

BRASIL. Ministério do Trabalho e Emprego. NR 06 - Equipamento de proteção individual - EPI. Brasília: Ministério do Trabalho e Emprego, 2009.

BREWCZYŃSKA, A. et al. The influence of the workplace-related biological agents on the immune systems of emergency medical personnel. Central European Journal of Immunology, v. 40, n. 2, p. 243-8, 2015.

DAL PAI, D. et al. Equipes e condições de trabalho nos serviços de atendimento pré-hospitalar móvel: revisão integrativa. Revista Eletrônica de Enfermagem, v. 17, n. 4, p. 1-12, 2015.

GUIMARAES, A. L. O.; FELLI, V. E. A. Notificação de problemas de saúde em trabalhadores de enfermagem de hospitais universitário. Revista Brasileira de Enfermagem, v. 69, n. 3, 2016.

LEITE, H. D. C. S. et al. Risco ocupacional entre profissionais de saúde do serviço de atendimento móvel de urgência-SAMU. Enfermagem em Foco, v. 7, n. 3/4, p. 31-35, 2016.

LORO, M. M. et al. Desvelando situações de risco no contexto de trabalho da Enfermagem em serviços de urgência e emergência. Escola Anna Nery Revista de Enfermagem, v. 20, n. 4, 2016.

MARTINS, K. M. et al. Accidentes con material biológico en los servicios de urgencia y emergencia. Ciencia y enfermería, v. 20, n. 2, p. 65-71, 2014.

OLIVEIRA, A.C.; MACHADO, B. C. A.; GAMA, C. S. Acidente Ocupacional envolvendo material biológico entre bombeiros militares de Minas Gerais.

Ciência Cuidado e Saúde, v.13, n.2, p.343-349, 2014.

OLIVEIRA, A. C.; PAIVA, M. H. R. S. Análise dos acidentes ocupacionais com material biológico entre profissionais em serviços de atendimento pré- 
hospitalar. Revista Latino-Americana de Enfermagem, v. 21, n. 1, p. 309315, 2013.

OLIVEIRA, Q.B.; SANTOS, R.S.; SANTOS, C.M.F. Acidentes de trabalho na equipe de enfermagem: uma revisão de literatura. Revista Enfermagem Contemporânea, v.2, n.1, 2013.

PIMENTA, F. R. et al. Atendimento e seguimento clínico especializado de profissionais de enfermagem acidentados com material biológico. Revista da Escola de Enfermagem da USP, v. 47, n. 1, p. 198-204, 2013.

RUFATTO, A. M. O uso de equipamentos de proteção individual pelos Bombeiros Militares do Estado de Santa Catarina no atendimento préhospitalar. Trabalho de Conclusão de Curso, 2011.

SOCIEDADE BRASILEIRA DE IMUNIZAÇÕES (SBIn). Calendário de Vacinação Ocupacional2018-2019, 2018.

SOERENSEN, A.A. Acidentes ocupacionais com ênfase ao risco biológico em profissionais do Atendimento Pré-hospitalar Móvel. Dissertação. Escola de Enfermagem de Ribeirão Preto - Universidade de São Paulo. Ribeirão Preto, 2008.

SOUSA, A. T. O.; SOUZA, E. R.; COSTA, I. C. P. Riscos ocupacionais no atendimento pré-hospitalar móvel: produção científica em periódicos online. Revista Brasileira de Ciência da Saúde, v.18, n.2, p.167-174, 2014.

TRIPPLE,A. F. V., et al. Acidente com material biológico no atendimento préhospitalar móvel: realidade para trabalhadores de saúde e não-saúde. Revista Brasileira de Enfermagem, v.66, n. 3, p. 378-84, 2013. 